\title{
The Control of Population Tumor Cells via Compensatory Effect
}

\author{
Janar J. Yermekbayeva*, Beisenbi A.Mamyrbek, Arystan N. Omarov and Gulnar A. Abitova* \\ Department System analyses and control, L. N. Gumilyov Eurasian National University, 11 Alexander Pushkin St, Astana, Kazakhstan
}

Received: 10 Jul. 2013, Revised: 12 Oct. 2013, Accepted: 13 Oct. 2013

Published online: 1 Jul. 2014

\begin{abstract}
Mathematical models and simulation studies are powerful tools to investigate dynamic properties of complex systems. Complex models with constant parameters often approach a steady state. In this research, we present an overview of mathematical approaches applied to the description of control population tumor cells. We are researching the influence of D-factor on tumor cells population. We called it compensatory effect. There is Vaccination as biological analogue of the compensator effect. D-factor is presented as catastrophe theory's fold form. Further, numeric results of model experiments are showing that this approach is positive efficient.
\end{abstract}

Keywords: Regulation of immune system, control system, robust stability, Lyapunov function, compensator effect, D-factor, structurally-steady mappings

\section{Introduction}

Today the control of factors, which influence the risk of oncological diseases, often attracts a constant interest.

At the moment, control factors researches influencing oncological diseases are very important. Modern medicine is providing reliable basis for screening, diagnosis, treatment, prevention and early intervention of cancer. Fortunately, it's level is high enough, so many cancer types are curable and in study process. But today problems of control complex mathematical models have not yet been sufficiently studied.

In this work we study the model of tumor growth. The model takes into account the interaction between the tumor cells and the two types of cells, regulating organism's antitumor activity.

We analysed and made the review of the previous works.

These scientists are: G.I.Marchuk, V.I.Kant, A.A.Klementyev, B.G.Ilyassov, L.M.Bakussov, L.Y.Klepper, V.A. Klimanov, V.Moiseenko, N.M.Emmanuelle, A.D.Bazykyn and other have studied and developed different mathematical models and methods of the study of medico-biological systems.

Khlebopros R.G. has proposed an effect of disciplined authophagy: the affected organ is removed from the organism and is placed into a special device for the perfusion of the isolated organs.

Khlebopros R.G., Sukhovolsky V.G., Slepkov V.A. studied the modeling of the growth and formation tumors.

In work [1] authors consider a nonlinear mathematical model of hematopoietic stem cell dynamics, in which proliferation and apoptosis are controlled by growth factor concentrations.

In paper [2,3] is a development a model for describing the dynamics of treated chronic myelogenous leukemia. This model is based on replacing the recent agent-based model.

On [4] research experimental systems demonstrated the model which consistently explains a broad variety in patients with chronic myeloid leukemia (CML).

J.Mann and M.Roberts in paper [5] discussed and incorporated the effect of vaccination into the model, by changing the initial susceptible population after each epidemic generation.

Thus the research in this paper (development the control system of tumor cells) is very important. It is represented interestingly not only as a basis for the solution of various applied tasks, but also as independent theoretical research.

\footnotetext{
*Corresponding author e-mail: erjanar@mail.ru, gulya.abitova@gmail.com
} 
Creation of control systems with control laws in a class of structural-steady mapping on the basis of two directions wasn't studied before.

The Multidisciplinary of two scientific ways will allow one to find new methods in the theory of control in immunology.

Thus, for the solution of the tasks classical and modern theory methods and control, theoretical and applied methods theory of dynamic systems, the modern theory of catastrophe and their application on population models will be used.

From our point of view the disease as outbreak (virus) of tumor cells on an organism. Our task is to suppress disease and get a stable state of an organism (influence vaccine).

\section{Mathematical Model}

We investigated the model of population cells and their interaction and influence.

The initial model, thus, includes three dynamical variables. This model can be simplified, keeping two of the variables- the concentration of tumor cells and that of the cells of specific resistance. The activity level of the cells of natural resistance is included as a parameter.

The differential equation model used to describe this system [5]:

$$
\begin{aligned}
& \dot{x}_{1}=j-\beta x_{1} x_{2}-\gamma x_{1}+\frac{\alpha x_{1} x_{2}}{1+x_{2}} \\
& \dot{x}_{2}=x_{2}-\frac{\mu x_{2}}{1+\delta x_{2}}-x_{1} x_{2} .
\end{aligned}
$$

Here $x_{1}$ is the concentration of the free (i.e. not connected with target cells) cells of specific resistance (effector cells) and $x_{2}$ is the concentration of tumor cells. Refer to Figure 1 for the phase portrait [6].

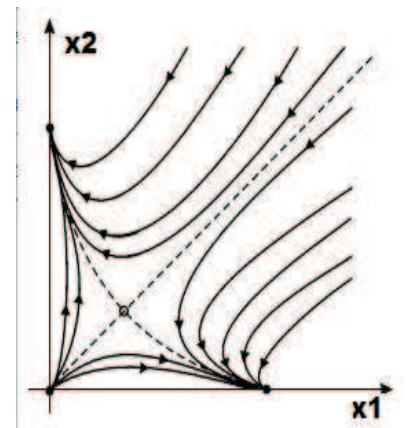

Fig. 1: Phase portrait of system 1

The model takes into account the following processes: $x_{1}$ - is the concentration of the free (i.e. not connected with target cells) cells of specific resistance (effector cells); $x_{2}$ - is the concentration of tumor cells;

$J$ - the effector cells' growth with velocity;

$\beta x_{1} x_{2}\left(x_{1} x_{2}\right)$-death effector and tumor cells as a result of their interaction;

$\alpha$ - the effector cells' multiplication and the concentration of tumor cells;

$\delta$ - the tumor cells' growth;

$\eta(\gamma)$ - natural death of tumor (effector) cells.

Thus we have system of two differential equations, depending on six parameters.

The stationary points of the system are determined from conditions $x_{1}=0$ and $x_{2}=0$ and the system 1 has three states of equilibrium:

$$
x_{s 1}=(0,0), x_{s 2}=\left(0, \frac{\eta-1}{\delta}\right), x_{s 3}=\left(\frac{j}{\gamma}, 0\right)
$$

Furthermore, the Jacobi matrix of the system 1 is

$$
J=\left[\begin{array}{cc}
\frac{\alpha x_{2}}{x_{2}+1}-\beta x_{2}-\gamma \frac{\alpha x_{1}\left(2 x_{2}+1\right)}{\left(1+x_{2}\right)^{2}}-\beta x_{1} \\
-x_{2} & 1-\frac{\eta}{\left(\delta x_{2}+1\right)^{2}}-x_{1}
\end{array}\right]
$$

In this work we propose a new method of controlling population interaction of the effector and tumor cells. This approach can create stabilization of the organism's state on a predictable level. We call this approach compensatory effect.

With the help of regulating factors, it is also possible to evaluate the control level of the cells's numbers. Let us add to the system 1 asynchronically controlling D-factor, represented as a function of structurally- steady mapping known as fold catastrophe.

One of the most efficient methods of analyzing non-linear systems is the bifurcation analysis. This is the process of determining the parameters change on system's behavior.

\section{Discussion}

In this paper we present an extension of the model of 1 , in which we made two major changes:

First, addition D-factor (as Vaccination);

Second, studying the stability state model from construct Lyapunov functions.

\subsection{The Influence control law on dynamic model}

We add the influence forms of one-parameter mappings to the right parts. One of the most important points of catastrophe theory is the behaviour of smooth functions, depending on a parameter.

The properties of these functions are determined by a number of results from functional analysis: Implicit function theorem, Morse lemma and Thom's theorem [7, 8]. 
Thus, the system obtained is the following:

$$
\begin{aligned}
& \dot{x}_{1}=j-\beta x_{1} x_{2}-\gamma x_{1}+\frac{\alpha x_{1} x_{2}}{1+x_{2}}-\left(x_{1}^{3}+k_{1} x_{1}\right) \\
& \dot{x}_{2}=x_{2}-\frac{\mu x_{2}}{1+\delta x_{2}}-x_{1} x_{2}-\left(x_{2}^{3}+k_{1} x_{2}\right)
\end{aligned}
$$

$D$-factor is presents in a view fold form (catastrophe form). Negative values mean the specific influence on organism and parameter $k_{1}$ is control value. The controlling parameter can be thought of as the influence of the therapy (Vaccination) being received.

The stationary points are determined from conditions $x=0$ and, therefore, the system 2 has states of equilibrium:

$$
\begin{aligned}
& x_{s 1}=(0,0), x_{s 2}=(M, 0), \\
& M=A-B, A=\sqrt[3]{\frac{J}{2}+\sqrt{Q}}, \\
& B=\frac{\gamma+k_{1}}{3 A}, Q=\left(\frac{\gamma+k_{1}}{3}\right)^{3}+\left(\frac{j}{2}\right)^{2}
\end{aligned}
$$

2.

The phase portrait of the system 2 is shown in Figure a

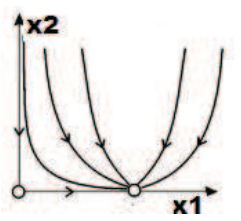

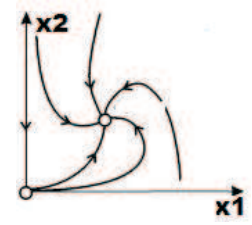

b
Fig. 2: Phase portait of the system

The Jacobi matrix of the system 2 is

$$
\begin{aligned}
J=\left[\begin{array}{l}
\frac{\alpha x_{2}}{x_{2}+1}-\beta x_{2}-\gamma-3 x_{1}^{2}-k_{1} \\
-x_{2}
\end{array}\right. & \left.\begin{array}{r}
\frac{\alpha x_{1}\left(2 x_{2}+1\right)}{\left(1+x_{2}\right)^{2}}-\beta x_{1} \\
1-\frac{\eta}{\left(\delta x_{2}+1\right)^{2}}-x_{1}-3 x_{2}^{2}-k_{1}
\end{array}\right]
\end{aligned}
$$

Stability is a fundamental notion in the qualitative theory of differential equations and is essential for many applications.

In turn, Lyapunov functions are basic instruments for studying the phenomenon of stability. Not only do they yield necessary and sufficient conditions for stability, but also help to solve a series of complex and subtle problems.

There is no universal method for the construction of Lyapunov functions.

However, in some special cases the construction is possible through some special techniques [9].

Let us study the system with non-linear equations 2 and define the conditions, at which here exist a region of stability (we construct the Lyapunov function).

We use the central Morse theorem with fold catastrophe from the classification of Thom's theorem.

\subsection{The method of construct Lyapunov function}

Let us construct Lyapunov function for system 2 and find the region of stability.

From the geometrical interpretation view the method of research stability state is reduced to creation of family the closed surfaces surrounding, which the beginning of coordinates. System state moves across contour curves.

The properties are integrated curves can cross each of these surfaces. The geometric meaning of a Lyapunov function used for determining the system stability about the zero equilibrium.

Here, we must to find Lyapunov's function, which is positive definite state function $V(x)>0$ and the time derivative is locally negative definite $\dot{V}(x)$ or $\dot{V}(x)=\frac{\partial V(x)}{\partial x} \frac{d x}{d t}<0$.

As is known from theory, given a system, described by equations of dynamics:

$$
\frac{d x_{i}}{d t}=\Phi_{i}\left(x_{1}, x_{2}, \ldots, x_{n}\right), \quad i=1,2, \ldots, n .
$$

the time derivative of the corresponding Lyapunov function has the form

$$
\frac{d V}{d t}=\frac{\partial V}{\partial x_{1}} \frac{d x_{1}}{d t}+\frac{\partial V}{\partial x_{2}} \frac{d x_{2}}{d t}+\ldots+\frac{\partial V}{\partial x_{n}} \frac{d x_{n}}{d t}
$$

or

$$
\frac{d V}{d t}=\frac{\partial V}{\partial x_{1}} \Phi_{1}+\frac{\partial V}{\partial x_{2}} \Phi_{2}+\ldots+\frac{\partial V}{\partial x_{n}} \Phi_{n}
$$

The gradient of a function $V\left(x_{1}, x_{2}, \ldots, x_{n}\right)$ is a vector, defined as

$$
\operatorname{grad} V=\left\{\frac{\partial V}{\partial x_{1}}, \frac{\partial V}{\partial x_{2}}, \ldots, \frac{\partial V}{\partial x_{n}}\right\} .
$$

One can introduce a vector $\Phi\left(x_{1}, x_{2}, \ldots, x_{n}\right)$ with projections, corresponding to 3 , namely

$$
\left\{\Phi_{1}=\frac{d x_{1}}{d t} ; \Phi_{2}=\frac{d x_{2}}{d t} ; \ldots ; \Phi_{n}=\frac{d x_{n}}{d t}\right\} .
$$

Vector $\Phi\left(x_{1}, x_{2}, \ldots, x_{n}\right)$ is a vector of velocity of a point $M$, depicted in Figure 3.

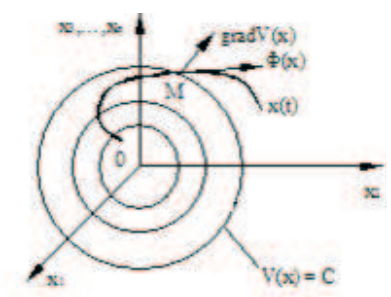

Fig. 3: The geometric meaning of a Lyapunov function

According to 4-5 one has 


$$
\frac{d V}{d t}=W(x)=\operatorname{grad} V \cdot \Phi(x)
$$

where $x_{1}, x_{2}, \ldots, x_{n}$. In other words, the derivative of the Lyapunov function, corresponding to the system, is a scalar product of the gradient of the function and the vector of phase speed.

Vector $\operatorname{grad} V(x)$ is perpendicular to the surface $V(x)=$ const and is directed towards $V(x)$ 's increase.

If $\frac{d V}{d t}<0$ then the angle between $\operatorname{grad} V(x)$ and $\Phi(x)$ is obtuse, i.e. strictly between 90 and 180 degrees, and the phase trajectory goes towards $V(x)$ 's decrease.

If both vectors $\bar{a}\left(x_{1}^{*}, y_{1}^{*}\right)$ and $\bar{b}\left(x_{1}^{*}, y_{1}^{*}\right)$ are non-zero, then the cosine of the angle between them is calculated by

$$
\cos (\bar{a} \wedge \bar{b})=\frac{x_{1}^{*} x_{2}^{*}+y_{1}^{*} y_{2}^{*}}{\sqrt{x_{1}^{* 2}+y_{1}^{* 2}} \sqrt{x_{2}^{* 2}+y_{2}^{* 2}}} .
$$

Let us split the model 2 and for the determination of stability construct the Lyapunov function.

We rewrite the system 2 as follows:

$$
\begin{aligned}
& \left(\frac{d x_{1}}{d t}\right)_{1}=-x_{1}^{3}-\left(k_{1}+\gamma\right) x_{1}+j \\
& \left(\frac{d x_{1}}{d t}\right)_{2}=\left(1-k_{2}\right) x_{2}-x_{2}^{3}
\end{aligned}
$$

For the right part (side) of 6 we take a vector-function, where the gradient is equal by absolute value and opposite in direction to the vector of speed of the system 2 (see Figure 4a).

And for the left part is chosen so that the angle between the gradient and the vector of phase speed is greater than 90 degrees (see Figure 4b).
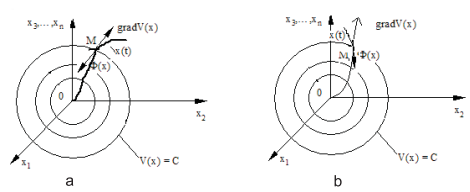

Fig. 4: The direction of vectors

The right part of 6 thus becomes

$$
\frac{\partial V_{1}}{\partial x_{1}} \frac{d x_{1}}{d t}=-\left(\frac{d x_{1}}{d t}\right)^{2}
$$

and

$$
\frac{\partial V_{1}}{\partial x_{2}} \frac{d x_{2}}{d t}=-\left(\frac{d x_{2}}{d t}\right)^{2}
$$

In order to find the remaining part of the Lyapunov function, we represent the vector of speed as

$$
x_{1}^{*}=\left(\frac{\alpha x_{1} x_{2}}{1+x_{2}}-\beta x_{1} x_{2}\right)
$$

and

$$
y_{1}^{*}=\left(-x_{1} x_{2}-\frac{\eta x_{2}}{1+\delta x_{2}}\right)
$$
$y_{2}^{*}=1$

The gradient function is chosen so that $x_{2}^{*}=-1 / \beta$ and

The numerator of the fraction when calculating the angle

$$
x_{1}^{*} x_{2}^{*}+y_{1}^{*} y_{2}^{*}=-\frac{\frac{\alpha}{\beta} x_{1} x_{2}}{1+x_{2}}-\frac{\eta x_{2}}{1+\delta x_{2}}<0
$$

By the physical meaning of the system 2, all the parameters and variables of the system are always positive.

Let us, write down the components of the Lyapunov function for the right part:

$$
\begin{aligned}
& \left(\frac{d x_{2}}{d t}\right)_{1}=\frac{\alpha x_{1} x_{2}}{1+x_{2}}-\beta x_{1} x_{2} \\
& \left(\frac{d x_{2}}{d t}\right)_{2}=-x_{1} x_{2}-\frac{\eta x_{2}}{1+\delta x_{2}}
\end{aligned}
$$

$$
\frac{\partial V_{1}}{\partial x}=\left[x_{1}^{3}+\left(k_{1}+\gamma\right) x_{1}+j ;\left(k_{1}-1\right) x_{2}+x_{2}^{3}\right]
$$

For the second part one has

$$
\frac{\partial V_{2}}{\partial x}=\left[-\frac{1}{\beta} ; 1\right]
$$

Lyapunov functions proper are obtained by integrating 9 and 10 by the variable $x$ :

$$
V_{1}=\frac{x_{1}^{4}}{4}+\frac{\left(k_{1}+\gamma\right) x_{1}^{2}}{2}-j x_{1}+\frac{x_{2}^{4}}{4}+\frac{\left(k_{1}-1\right) x_{2}^{2}}{2}
$$

From 11 positive definiteness at the origin is not immediately apparent. Therefore one can use Morse's lemma from catastrophe theory. The function 11 satisfies the conditions of the lemma.

Let us find the Hessian matrix (the stability matrix) at the origin only for $V_{1}$ since the matrix for $V_{2}$ becomes zero:

$$
\left.\frac{\partial^{2} V_{1}(x)}{\partial x_{1} \partial x_{1}}\right|_{0}=3 x_{1}^{2}+k_{1}+\left.\gamma\right|_{0}=k_{1}+\gamma
$$

$$
\begin{gathered}
\left.\frac{\partial^{2} V_{1}(x)}{\partial x_{2} \partial x_{2}}\right|_{0}=k_{1}-1-\left.3 x_{2}^{2}\right|_{0}=k_{1}-1 \\
\left.\frac{\partial^{2} V_{1}(x)}{\partial x_{1} \partial x_{2}}\right|_{0}=\left.\frac{\partial^{2} V_{1}(x)}{\partial x_{2} \partial x_{1}}\right|_{0}=0 ;
\end{gathered}
$$

$V(x)=\left\|\begin{array}{cc}k_{1}+\gamma & 0 \\ 0 & k_{1}-1\end{array}\right\|=\left(k_{1}+\gamma\right) x_{1}^{1}+\left(k_{1}-1\right) x_{2}^{2}$

Positive definiteness follows from

$$
\left\{k_{1}+\gamma\right\}>0 ; k_{1}>1
$$


We proved that our Lyapunov function is positive definite 13 and the time derivative is negative definite 7 . Thus, the found factors define area of robust stability state system 2 .

Geometrical interpretation of Lyapunov function and results of application Morse lemma from the catastrophe theory is considered $[10,11]$.

\subsection{Model experiments}

Let's give some experimental data of the described models.

1.Model 1 on parameters

$$
J=10, \beta=1.3, \alpha=3, \eta=0.4, \delta=0.04, \gamma=5
$$

Result in Figure 1.

2.Model 2 on parameters

$$
J=10, \beta=1.3, \alpha=3, \eta=0.4, \delta=0.04, \gamma=5,
$$

$k_{1}=1.5$ Result in Figure 2a.

3.Model 2 on parameters

$$
\begin{aligned}
& J=0.35, \beta=1.42, \alpha=2.025, \eta=0.008, \delta=0.8 \\
& \gamma=1.6, k_{1}=0.5
\end{aligned}
$$

also view on Figure 2b. The stationary point $(x=0.16, y=0.58)$

4.Model 2 on parameters

$$
\begin{aligned}
& J=0.35, \beta=1.42, \alpha=2.025, \eta=0.008, \delta=0.8, \\
& \text { gamma }=1.6, k_{1}=-3.5
\end{aligned}
$$

also view on Figure 2b. The stationary point $(x=0.96, y=1.86)$

5.Model 2 on parameters

$$
\begin{aligned}
& J=10, \beta=1.42, \alpha=2.025, \eta=0.008, \\
& \delta=0.8, \gamma=1.6, k_{1}=-3.5
\end{aligned}
$$

also view on Figure 2b. The stationary point $(x=2.32, y=1.47)$

\subsection{Results}

In this paper we research population of tumor cells.

The description of parameters was presented. Stationary points are found. Then adds the influence forms of one-parameter mappings to the right parts (D-factor).

Find states the equilibrium and phase portrait of the system. After construct Lyapunov function for system 2 and find the region of stability.

Thus, we have obtained a Lyapunov function with negative time derivative and conditions of the Hessian's positive definiteness.
In physical terms this means, that the controlling parameter somewhat decreases the effector cells' population.

The biological meaning is, that in order for the system to be stable, the growth of the effector cells $(J)$ must be positive, i.e. there should be enough protein in the cells.

The parameters of interaction natural mortality rate $\gamma$ and $k_{1}$ of the controlling are essential. The interaction between the tumor and the effector cells leads to the (negligible) decrease of the latter; in contrast, the former decreases considerably, up to the total extinction when subject to special medical treatment.

So, the interaction between the tumor and the effector cells leads to the (negligible) decrease of the effector cages. And also tumor cells decrease after influence the special medical treatment.

The parameters of the effector cells, therefore, have to be chemo-stable. Influence of fold form the catastrophe theory on model 1 has positive result. Some experimental data of the described models were carried out.

Thereby we obviously operate of population tumor cells number.

In this paper we research a method of robust stability (compensatory effect) which can be applied to control of growth tumor cells in an organism.

Results of this work can be used the development of new theoretical method for stabilization immune system at predicted level $[12,13]$.

\section{Conclusion}

Structurally-steady mapping is one of the methods of controlling the growth of the tumor cells. The character of phase portraits shows two states: the first state (Fig. 2) with zero concentration of the tumor cells, i.e. the complete convalescence.

Second state is transfer from tumor state to a sleeping state, i.e. growth control at the expense of strengthening effector cells (immune system).

In this work the model of dynamics tumour's growth taking into account interaction with two types and steady mapping from the theory of catastrophe is considered.

The main result is the synergistic approach compensatory effect which can be used to control the growth of tumor cells, and fixes the immune system on a predictable level. One can evaluate the level of controllability among the tumor cells number with the help of regulating factors.

Finally, problems of control and impact factors on development risk oncological diseases are caused constantly by a great interest.

Many aspects of interaction specific immune forces and malignant education can be found out during research.

In future we will investigate a boomerang-effect at artificial decrease in number cooperating populations and find other method for construct Lyapunov function. 
Research of idea and methods synergetic systems is offered. This results is particularly relevant, as new method in mathematical science, new direction of theory catastrophe and their applied views. The concept creation of a control system dynamic objects with the increased potential with robust stability.

The offered results can be used at theoretical studies influencing the medicobiological preparations on tumor cells. Also for identifications of control ways on oncopatology and regulation of immune system an organism.

\section{Acknowledgement}

Authors would like to thank L.N.Gumilyov Eurasian National University for funding this project (under Grant No.264).

\section{References}

[1] M. Adimya, F.Crauste. Mathematical model of hematopoiesis dynamics with growth factor-dependent apoptosis and proliferation regulations. Mathematical and Computer Modelling, 49, 2128-2137 (2009).

[2] Peter S. Kim, Peter P. Lee and Doron Levy. Modeling Imatinib-Treated Chronic Myelogenous Leukemia: Reducing the Complexity of Agent-Based Models. Bulletin of Mathematical Biology, 70, (2008).

[3] Peter S. Kim, Peter P. Lee and Doron Levy. A PDE Model for Imatinib-Treated Chronic Myelogenous Leukemia. Bulletin of Mathematical Biology, 70, (2008).

[4] Ingo Roeder, Maria Herberg and Matthias Horn. An AgeStructured Model of Hematopoietic Stem Cell Organization with Application to Chronic Myeloid Leukemia. Bulletin of Mathematical Biology, 71, (2009).

[5] A. D. Bazykyn, Nonlinear dynamics of interaction of population, Moscow, (2003).

[6] R. G. Khlebopros, V. A. Okhonin, A. I. Fet, Catastrophes in Nature and Society, 1, (2010).

[7] G. Nikolis, I. Prigogine, Exploring complexity: an introduction, Moscow, (1990).

[8] T. Poston, I. Stewart, Catastrophe Theory and its Applications, Dover Publications, (1996).

[9] M. A. Beisenbi, K. S. Kulniyazova. Determining the region of robus stability of control systems via Lyapunov functions, in: The proceedings of the VIth international asian schoolseminar 2010.SB RAS Novosibirsk.

[10] J. J. Yermekbayeva, A. N. Omarov, Compensatory effect for controlling the population of phytophages and enthomophages, Eurasian Enthomological Journal, 10, (2010).

[11] J. J. Yermekbayeva,The doctoral thesis. The analysis and research of complex behaviour population models. ISBN 9965-31-334-4, L. N.Gumilyov Eurasian National University, Reviewer: R.G.Khlebopros, (2010).
[12] J. J. Yermekbayeva, Control of tumoral cages growth with the help of "compensatory effect". The proceedings of the XVI All-Russia symposium with international participation Complex systems in extreme conditions, Krasnoyarsk scientific center of the Siberian Branch of the Russian Academy of Science, (2012).

[13] G. Abitova, V. Nikulin, M. Beisenbi, A. Ainagulova. Control System with High Robust Stability Characteristics Based On Catastrophe Function. The proceedings of the ICECCS', 12, (2012).

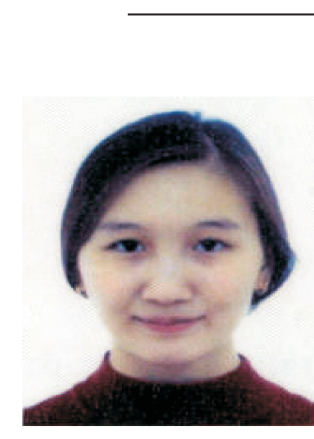

Janar Yermekbayeva is a Leading Researcher, $\mathrm{PhD}$, Lecturer of Department System analyses

and control L.N.Gumilyov Eurasian National University, she has published more than 30 research articles in USA, Kazakhstan, Russian Federation, Malaysia, Poland.

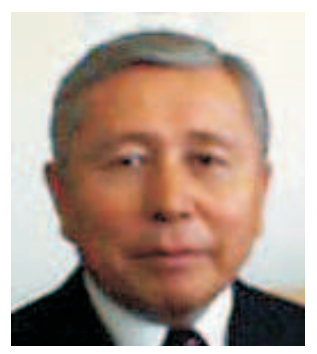

Beisenbi Mamyrbek is a leading world-known figure in automatics and is presently employed as Professor at ENU, Kazakhstan and Russian. He obtained his $\mathrm{PhD}$ from Bauman Moscow State Technical University. He introduced a new technique, now called as Methods of increase the capacity of robust stability control systems. $\mathrm{He}$ has been personally instrumental in establishing "Automation and Control" $\mathrm{PhD} / \mathrm{MS} / \mathrm{BSc}$ programs at ENU. He has been an invited speaker of conferences and has published more than 200 research articles in reputed international journals of mathematical and engineering sciences.

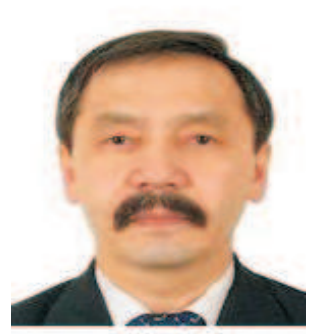

Arystan

Omarov is a Associate. Prof, $\mathrm{PhD}$, Lecturer of L.N.Gumilyov Eurasian National University, he has published 70 research articles. He obtained his PhD from Krasnoyarsk scientific center of the Siberian Branch, Russian Academy of Science. 


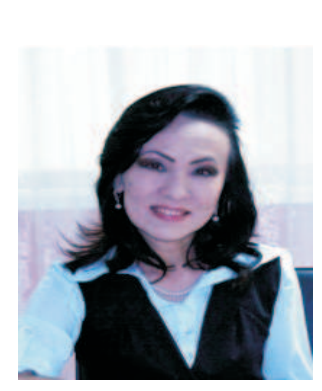

Gulnar Abitova
is a Associate $\begin{array}{r}\text { Prof, PhD, } \\ \text { Leading Researcher, } \\ \text { Lecturer of Gumilyov } \\ \text { ENU. She has been an invited } \\ \text { speaker of conferences } \\ \text { and has published more than } \\ 30 \text { research articles in reputed } \\ \text { international proccesings of } \\ \text { mathematical and engineering } \\ \text { sciences in the USA, Canada, }\end{array}$

France, Turkey, Malaysia, Bulgary, Hungary. Her papers became a basis for a chapter of book in Springer, Scopus. She served as a Track Chair of the Conference - IEOM 2012 Istanbul, Turkey. 